\title{
Climate Information: Towards Transparent Distillation
}

\author{
Christopher D. Jack, John Marsham, David P. Rowell, \\ and Richard G. Jones
}

\begin{abstract}
Constructing climate information to inform climate change risk-related decision-making is challenging and requires a rigorous interrogation and understanding of multiple lines of evidence and an assessment of the values, limits and uncertainties involved. Critically, there is no definitive approach agreed on by all climate scientists. Rather, a range of approaches and assumptions are used, with implications for robustness,
\end{abstract}

C. D. Jack $(\bowtie)$

Climate System Analysis Group, University of Cape Town,

Cape Town, South Africa

e-mail: cjack@csag.uct.ac.za

J. Marsham

University of Leeds, Leeds, UK

D. P. Rowell

Met Office Hadley Centre, Exeter, UK

R. G. Jones

Met Office Hadley Centre, Exeter, UK

Oxford University School of Geography and the Environment, Oxford, UK

(C) The Author(s) 2021

D. Conway, K. Vincent (eds.), Climate Risk in Africa, https://doi.org/10.1007/978-3-030-61160-6_2 
reliability and uncertainty. Often these choices and assumptions are informed by the values and objectives of climate science rather than the decision context. We propose an approach, information distillation, that makes explicit and open for deliberation many of the implicit decisions and value judgements that occur throughout the process of constructing information. We argue that this approach must engage substantively with the decision context and open up choices and assumptions in a transparent manner to deliberation across climate scientists and context experts. This should ensure relevance and usability, and build understanding and trust to form an important basis for effective uptake of information. Two case studies are described demonstrating the effectiveness of these approaches and illustrating several important principles for transparent information distillation.

Keywords Information distillation • FRACTAL • HyCRISTAL • Co-production • Uncertainty

\section{INTRODUCTION}

Responding effectively to the complex challenge of climate change requires well-informed decision-making. Climate science is crucial to the process, for describing and understanding past changes and projecting possible changes in the future, under different greenhouse gas emission scenarios. However, climate science involves many value judgements and choices, with consequences for the resultant information, decision-makers and stakeholders. Therefore, robust decision-making in the face of uncertainties requires transparent interrogation and deliberation of values and choices in order to distil reliable information and manage risk.

Scientific understanding of climate change globally and for Africa has progressed significantly over recent decades. Two key drivers of progress have been improvements in observations and modelling. First, new observational platforms, primarily satellite based, have generated an unprecedented volume of data on atmospheric, land surface and ocean variables. Regrettably, however, the availability of primary surface observations from weather stations is declining in many countries, particularly in Africa. Satellite observations are not enough on their own-they generally require 
cross-checking (calibration) against surface observations and for some variables such as near surface air temperature, obtaining accurate satellite estimates is challenging (Hooker et al. 2018).

Second, climate model complexity has advanced with the inclusion of a greater number and more realistic representations of climate processes. Global Climate Models (GCMs) included in the first Coupled Model Intercomparison Project (CMIP) that provided key evidence in the Intergovernmental Panel on Climate Change (IPCC) First Assessment Report (1990) were far simpler than the GCMs contributing to CMIP6 and the ongoing IPCC Sixth Assessment Report. For example, the GCMs participating in CMIP6 are predominantly Earth Systems Models (ESMs) which include coupling of models of the atmosphere and its chemistry, the oceans and their biology, sea-ice, and the land surface vegetation, with grid spacings generally in the range of 100-200 km (Eyring et al. 2016). Moreover, advances in computational capacity and scientific understanding have enabled the latest limited area Regional Climate Models (RCMs) to run with grid spacings of $4 \mathrm{~km}$ or less, allowing them to resolve features at spatial scales of around $25 \mathrm{~km}$, and explicitly capture deep convectiona key feature of climate in Africa. Such models can reproduce realistic convection, one of the long-standing challenges of climate modelling (Stratton et al. 2018; Kendon et al. 2019; Jackson et al. 2020).

These major advances in climate science through observations and modelling provide increasingly robust evidence that the climate is changing and that mitigation is crucial for constraining the extent of future climate change and associated impacts. However, there remain significant barriers to providing climate information in the format and level of accuracy often desired to support local-scale adaptation decision-making. Indeed, participants at the lst African Climate Risks Conference (ACRC 2019) emphasised the need to better integrate climate science research into decision-making, while noting the ongoing challenge that uncertainty in climate projections represents to this goal.

This challenge is being approached from two related perspectives: Vincent et al. (Chap. 3) adopt the perspective of integrating climate information more effectively into decision-making through co-production, while this chapter considers the potential for climate science and modelling to provide more considered and defensible information. In the next section, we describe the basis for constructing climate information, highlighting the important concepts of robustness and reliability and the associated assumptions. In particular, we consider the role of value judgements 
in characterising and reducing uncertainty, and how trade-offs between different types of error arise. This leads into the following section where the concept of climate information distillation is introduced. Climate information distillation strives to facilitate greater transparency and inclusion of decision-makers and stakeholders in the value judgements and trade-offs that are generally only considered within the climate science. This is followed by two case studies of climate science information development designed to aid decision-making, supported through the Future Climate for Africa (FCFA) programme. We end with brief conclusions.

\section{Constructing Robust and Reliable Climate Information}

One of the most common requests from climate risk management and adaptation exercises is for robust and reliable climate information with low uncertainty. It is worthwhile to step back and consider these terms, how we understand them, and how they are crucial to support the improved use of climate information in decision-making. First, we will consider robustness, or the strength of the evidence behind the information. For climate projections, this relates to climate models and their complexity and realism, and how we evaluate their realism. We then consider the closely related concepts of reliability and uncertainty which relate to the possibility of error - understandably a key concern for decision-making.

\section{Robust Information}

The IPCC Fifth Assessment Report guidance note on uncertainties (Mastrandrea et al. 2011) describes robust messages as those supported by multiple, consistent and independent lines of high-quality evidence. Highquality evidence rests on strong and well-tested assumptions, rigorous analysis and statistical testing, and validation against observations. However, different lines of high-quality evidence can and do result in different conclusions. Evaluation of multiple lines of independently produced evidence and their agreement or disagreement provides a strong basis for establishing robustness.

Mastrandrea et al. (2011) also note that evaluation of the degree of robustness involves expert judgement. For example, assessment of historical trends of extreme rainfall events over Africa are often reliant on 
spatially and temporally sparse weather station data with uncertain quality control. Even where multiple independent analyses exist and are consistent (e.g. from different gridded datasets of temperature and rainfall in Africa), they might all assume that the underlying primary data are reliable enough to draw conclusions. Whether this is indeed the case can often not be objectively determined but requires a level of expert judgement which is subjective and may vary from one climate scientist to another.

Establishing robustness of information based on future climate projections is particularly challenging to determine because, as we will discuss later, we cannot verify projections of the future. In this case, robustness rests on three bases:

1. Comprehensive representation of physical process understanding in models: Current understanding of climate system dynamics and feedbacks is both informed by and informs model development. We know that models must realistically represent the fundamental dynamics as well as important physical processes such as convection, land surface, ice-albedo and cloud feedbacks.

2. Ability to reproduce relevant aspects of historical climate variability: We cannot validate model simulations of future climate change, instead we validate their simulations of past climate modes of variability or trends that are deemed relevant to future projected changes.

3. Multi-model ensemble agreement: In line with the IPCC guidelines, if multiple models simulate the same future changes, then this provides multiple lines of evidence in support of a message. If multiple models diverge, they provide less support for any particular message. However, as will be discussed in the subsequent section on uncertainty, models are not completely independent and multimodel agreement is not a sufficient basis for robustness.

These three bases of robustness are closely interrelated. As models are developed in order to represent more processes, or improve their realism, model evaluations tell us how these developments affect the model's simulation of observed climate variability.

\section{Model Realism}

Model realism is advancing through two main avenues: improved realism of model components and parameterisations; and increased spatial 
resolution. The latter allows for more realistic representation of the land surface, including the crucial roles of topography, oceans and atmospheric convection. Until recently, higher resolution climate information has been restricted to RCMs or statistical downscaling (SD) to generate more local detail from coarser GCM grids. However, RCMs and SD are dependent on the realism of the driving GCM and it is not always clear under what conditions RCMs improve the realism of the climate simulation (Dosio et al. 2019). Among other limitations, SD methods also assume that observed climatological relationships will hold in the future warming world (statistical stationarity) which is not necessarily the case (Jack and Katragkou 2019). The added value or realism of regional modelling and $\mathrm{SD}$ is another area where multiple viewpoints are held, and expert judgement is deployed.

Computational capacity has now reached the point of enabling convection-permitting resolutions in atmospheric models-a major advance-because climate models generally use convection parameterisations that, while based on physical principles, are significant simplifications of reality and are a cause of much model disagreement or uncertainty (Sherwood et al. 2014). Explicitly permitting convection is an important advance in model realism and initial experiments have demonstrated significant improvements, particularly in the simulation of tropical convective rainfall characteristics over Africa (Stratton et al. 2018).

\section{Model Evaluation}

Guiding and understanding advances in climate model realism rests strongly on approaches to model evaluation. At the global scale, we have high confidence in the ability of contemporary climate models to reproduce observed global aggregate trends such as global mean near-surface air temperatures. Most models also realistically simulate responses to climate events such as large volcanic eruptions. Such comparisons have proven a mainstay for defending climate model realism.

At the regional to local scale, in the context of constructing information to inform decision-making, a key element of model evaluation is determining which climate features are relevant to robust future climate change projections. While there has been a call for greater focus on model evaluation in Africa and other regions (James et al. 2018), care must be taken to ensure that models are evaluated with respect to features across multiple scales that are likely to be relevant to future climate changes 
rather than just contemporary climate means and variability. Importantly, there has been a strong drive towards process-based evaluations which focus on key regional processes such as regional moisture transport dynamics, rather than surface variables (diagnostics) such as rainfall amounts. One framing developed under the Future Resilience for African Cities and Lands (FRACTAL) project is that of process chains which recognises and helps characterise the many interlinked processes that are relevant to climate change in a region (Daron et al. 2019). Another, used in the Integrating Hydro-Climate Science into Policy Decisions for ClimateResilient Infrastructure and Livelihoods in East Africa (HyCRISTAL) project, is a 'future-centric' approach to constraining the spread amongst model projections (Rowell 2019, and reference therein).

Advances in model complexity coupled to ongoing and more sophisticated process-based evaluations of model realism are critical foundations for the construction of robust climate information. However, even as the realism and performance of models have improved, the spread (or divergence) of projected changes from different models (referred to as multimodel ensembles) has often not reduced (Knutti and Sedláček 2013). Model advances, thus far, are not producing clear multi-model convergence in future projections, particularly of rainfall and for large parts of sub-Saharan Africa (see Chap. 1). Analysis of multi-model ensembles and characterization of uncertainty therefore remain critically important in constructing reliable messages about future climate change.

\section{Reliable Information and Reducing Uncertainty}

Unlike robustness, which relates to the characteristics of the evidence, reliability relates to the probability of error-a challenging concept for future climate projections. More formally, in weather and seasonal forecasts, reliability is one important measure of the track record of a forecast system over multiple forecasts. Reliability refers to the ability of the forecast system, over multiple forecasts, to reproduce the probabilities of climate variations. For example, forecasts of a $60 \%$ chance of dry conditions should, if perfectly reliable, be matched by actual dry conditions $60 \%$ of the time (Wilks 2011). Reliability of weather and seasonal forecasts can be evaluated retrospectively, but we do not have that luxury for climate change projections and therefore must rely on less empirical measures of reliability. In practice, decision-makers interpret reliability to mean that the information should not turn out to be wrong. 
There are fundamentally two ways that climate information can be wrong; we can fail to identify a climate future that does occur and so risk not planning adequately, or we can identify a future that does not occur and plan unnecessarily. It is conceptually simple to avoid the first type of error. We can present a very wide range of future changes and be highly confident that reality will lie somewhere inside the range. However, we then cannot avoid the second type of error as we are almost certainly identifying future climates that will not occur. This approach can force decisionmaking towards no-regrets approaches that while robust under any plausible future climate, can be very expensive (requiring finance to cover conditions that are never reached) and have other undesirable consequences. There is therefore a strong argument for and motivation to constrain the uncertainty range associated with multiple climate model projections and reduce the risk of identifying futures that are not going to occur (e.g. Chap. 6). This requires drawing on elements of robustness described previously to provide a basis for excluding some futures. However, first we need to understand and characterise different sources of uncertainty and the scope for reducing uncertainty.

\section{Sources of Uncertainty}

Any model, regardless of its complexity and resolution, or even its ability to reproduce past climate features, remains a simplified and imperfect representation of the real climate and so there is inherent uncertainty about any simulation of future climate. To characterise this uncertainty, ensembles of semi-independent models (Knutti et al. 2013) are used to construct a range of possible future changes. Models are only semi-independent because many models share core components and parameterisation schemes. The assumption being that a sufficiently large set of independent models will produce a range of future projected changes that approximates the actual uncertainty due to model weaknesses. The extent to which this is true is unclear and there are several associated concerns regarding model independence and their coverage of different processes that generate uncertainty (Parker 2013). Furthermore, where processes relevant to a particular regional climate change response, such as aerosol feedbacks, are inadequately represented in all models from an ensemble, it is possible that the ensemble range does not even include the real future (Rowell et al. 2015). 
Additionally, the climate system has an inherent stochastic component that we call natural variability. This means that any particular year, or decade, or even multiple decades can be warmer or cooler, wetter or drier, for no other reason than the interacting internal processes that generate semi-stochastic (or randomly determined) variability. Two to three decades into the future, natural variability is typically the largest source of uncertainty because other drivers, such as greenhouse gas concentrations, will be relatively small and difficult to distinguish from natural variability. Beyond one- or two-decades, changes in greenhouse gas concentrations relative to the present become much larger, depending on mitigation progress, and models project larger changes. Beyond around 50 years into the future, the proportion of uncertainty arising from unknown future greenhouse gas concentrations increases substantially (Hawkins and Sutton 2011).

\section{Reducing Uncertainty}

The uncertainty associated with natural variability is essentially irreducible. Uncertainty in emissions scenarios is not reducible through climate science; however, when constructing information to inform decisions, it should either be clear why particular emissions scenarios have been selected, or stakeholders should be involved in the selection. These choices often represent the value judgements of climate scientists rather than decision-makers.

A common approach to reducing model derived uncertainty is to decide which simulated futures are less plausible. Simple approaches to this involve discarding the most extreme changes by, for example, only presenting the 25th to 75 th percentile range (e.g. as done in the IPCC AR5 Atlas, Van Oldenborgh et al. 2013) under the assumption that more extreme changes are less plausible. However, there is very little basis for this and more defensible approaches involve evaluating model realism under historical climate conditions, with respect to variables or regional climate features of relevance to future regional climate change (e.g. $\mathrm{McSweeney} \mathrm{et} \mathrm{al.} \mathrm{2012).} \mathrm{Here,} \mathrm{process-based} \mathrm{model} \mathrm{evaluation,} \mathrm{described}$ earlier, can play an important role. For example, Rowell (2019) evaluates the realism of models in the CMIP5 ensemble with respect to observed important linkages between clouds and ocean temperatures in the southern Indian Ocean and regional rainfall. This observational constraint 
provides a basis for excluding one particular model and reducing the spread of projections by one third.

Models that fail to meet some subjective threshold of realism could be excluded from the ensemble. In some cases, these models turn out to be those projecting changes at the extremes of the multi-model range and their removal reduces the range. In southern Africa, for example, research shows models that simulate far too much rainfall in the current period project more drying (Munday and Washington 2019). If it is assumed that the error in current climate generates the high climate change, those models can be removed. In other cases, the excluded models are not outliers, and removing them does not greatly reduce the ensemble range (Rowell et al. 2016; Chap. 6).

It is important to note that there is no strong agreement about if and how model evaluation and exclusion is done. This is another area of expert judgement and diverse perspectives and values. There is some progress in identifying evaluation metrics that capture key features of climate in Africa (James et al. 2018) but approaches to model selection (and their effects on the model range) are still being explored, partly because the process involves numerous value judgements. We argue that the consequences of these choices can have significant implications and stakeholders and their values and expertise should be involved in making them. The next section describes emerging approaches to address this challenge.

\section{Climate Information Distillation}

Given the challenges and the advances described earlier, how do we make progress in constructing climate information to support decision-making? One framing of this construction process is increasingly called distillation. Though the interpretation of this phrase is varied, essentially the focus is on constructing information that is usable, robust, and reliable for decisionmaking (e.g. Giorgi 2020). Distillation involves identifying the value in or establishing the meaning of evidence. Because value and meaning are inherently contextual, distillation is necessarily deeply rooted in context. For example, the meaning of an ensemble of climate simulations may be very different for an urban planner and a climate modeller.

Co-production approaches (see Chap. 3) can be very effective in identifying the information needs of decision-makers and translating science into understandable and relevant information. However, within any information construction processes, whether through co-production or not, 
many decisions and value judgements are made related to realism, robustness, uncertainty, and the risk of making errors. In many cases, these decisions and judgements are made out of context, or with little consideration of context. For example, climate scientists frequently decide that reducing uncertainty is more valuable than the associated risk of failing to identify the real future climate. These critical value judgements are rarely made collaboratively with those managing the risks of error. The implications are seldom understood by either scientists or decision-makers.

We propose an approach to distillation that makes explicit and open for deliberation many of the implicit decisions and value judgements that occur throughout the process of constructing information. We argue that opening up these judgements and decisions through transparency and deliberation builds the critical trust and common understanding of the value (and the limits to value) climate science brings to a decision. Emerging learning (see Chaps. 3 and 7) suggests that these principles may be as important and potentially more valuable for integrating robust climate information into decision-making than efforts to simplify and communicate climate science outputs (Harold et al. 2019; multiple chapters in this volume) - that is, the process of engagement is as important as the climate information itself.

In the next section, we present examples of constructing climate information to support decision-making through the lenses of distillation, before we conclude with some principles and guidelines for distillation itself.

\section{Case STudies}

\section{FRACTAL}

The Future Resilience for African Cities and Lands (FRACTAL) project aimed to inform climate resilient decision-making in large capital cities in southern Africa. The project chose to adopt a strongly context-led approach to the development of climate information-one that incorporated and refined ideas about information distillation.

The context-led approach was integrated into the research design through pre-proposal consultation and motivated by prior experience in such projects. This was subsequently initiated by supporting city participants ranging from city councillors, urban planners, local academics, representatives of water utilities, power utilities, and civil society organisations 
(in particular informal settlement representatives) to collectively agree on a suite of "burning issues"-areas of significant common concern across all participants. In most cases, these emerged as insecure water supply, flooding, and sanitation, with a strong focus on peri-urban or informal housing areas. The burning issues were then unpacked progressively through a series of Learning Labs (McClure 2020), based on strong transdisciplinary principles, and are described more fully in Chaps. 3 and 7.

Of importance was the mode of introducing climate information in Learning Labs. In most of the formal interactions climate information was either not introduced in the first workshop activity, or if it was, it was only as a very small component. The objective was to avoid climate science (and climate scientists) strongly defining and framing the values and collaborative learning process.

When climate science information was introduced into the Learning Lab process, it was done through Climate Risk Narratives (Jack et al. 2020). These are descriptions of the city under different plausible (supported by scientific evidence) future climate conditions. In most cases, the initial narratives were informed by three plausible climate futures based on conventional analysis and interpretation of multi-model ensemble projections of climate variables and statistics perceived as relevant for the context.

Climate Risk Narratives became an iterative engagement device through subsequent Learning Lab workshops. Participants were involved in developing descriptions of the socio-economic elements of each narrative, which involved extensive deliberation over what the consequences of different climate futures would be, for whom and what responses were relevant. This prompted and allowed for a diversity of perspectives and values to be expressed. For example, in Windhoek, representatives from a youth organisation wanted the described futures to be optimistic and to reflect their aspirations for the successful implementation of the city's adaptation plans, rather than just negative challenging impacts.

Overall, distilling climate information that effectively engages with decisions is a process that involves building trust, agreeing on common values and priorities, integrating a diversity of experience, evidence, and expertise, and collaboratively managing risk and uncertainty. Reflecting on the process, three important aspects of climate risk narratives are particularly relevant to climate information distillation:

1. The first was the adoption of a risk framing. In Lusaka, the climate projections include large uncertainty about changes in rainfall, with 
projections spanning increasing and decreasing rainfall. However, discussions revealed that primary concerns were the impacts of rapidly increasing population and water demand, and reduced total rainfall but more intense rainfall events. Increases in total rainfall did not emerge as a concern. The narratives for Lusaka therefore do not represent a future with increasing rainfall-its exclusion was a collective decision made with consideration and understanding of the climate evidence-a value judgement that reflects the values and priorities of those making decisions or experiencing their consequences.

2. Secondly, very little time was spent on visualization, tailoring, or simplification of climate science evidence. Rather, climate scientists openly engaged with each other and with participants as decisions such as excluding particular futures were made. Building mutual understanding and trust in distillation decisions was prioritised over one-way modes of communication that may pre-emptively close down debate. This is not to devalue approaches to communication, which still have significant value and importance, but rather it is an argument for building trust in order to support legitimacy and collective ownership in the process (Harold et al. 2019).

3 . Finally, the perceived barrier of model-related uncertainty rapidly diminished in most cases. Once participants were able to engage with and build common understanding across the range of plausible futures, effective and priority interventions emerged, many of which were common across all plausible futures. In many cases, these interventions were based on good development and urban planning that would also be effective adaptation measures. While efforts to reduce model uncertainty are certainly valuable and climate science should and will continue to strive towards this, the perceived barrier of uncertainty is not always insurmountable, particularly where climate information is constructed through open, transparent, and collaborative distillation.

\section{HyCRISTAL}

The Integrating Hydro-Climate Science into Policy Decisions for ClimateResilient Infrastructure and Livelihoods in East Africa (HyCRISTAL) consortium, also part of the FCFA programme, focused on climate risk and advancing climate science in East Africa (Finney et al. 2019). 
Discussions with regional authorities and other stakeholders identified urban water sanitation and hygiene, and rural livelihoods as two important concerns for climate change-informed decision-making. These formed the subjects of pilot studies of decision-making within the project, alongside research on tea production, Lake Victoria water levels and water management.

HyCRISTAL's climate science addressed understanding of specific aspects of climate relevant to the aforementioned concerns, such as rainfall accumulations, extreme rainfall and rainy season onset. Drawing on this research, the wider literature, and discussions with stakeholders, HyCRISTAL also constructed Climate Risk Narratives (e.g. Burgin et al. 2019), with three possible futures spanning much of the plausible range in key variables of model projections, with the underpinning evidence provided in a technical appendix. This enabled HyCRISTAL to use the Climate Risk Narratives for engagement with decision-makers and planners, without conversations becoming too distracted or deterred by the scientific evidence. Moreover, the underpinning evidence was available for transparency, and for legacy so that the Climate Risk Narratives can easily be modified as the evidence base changes.

Climate Risk Narratives were generated for urban and rural contexts and were widely shared giving indicative impacts of each possible future. At forums such as the GHACOF (Greater Horn of Africa Climate Outlook Forum), they were found useful for engaging individuals in non-climate sectors. For particular decision contexts, HyCRISTAL also generated bespoke projections, for example, of possible changes in flood frequency in key cities, by (i) statistically downscaling CMIP models (unless the models had been shown to be implausible; Rowell 2019); (ii) using changes from state-of-the-art convection-permitting RCM simulations that explicitly model the rain-generating storms, thereby improving the representation of key processes and as a result giving larger changes in extremes than other model types (Kendon et al. 2019; Finney et al. 2020); and (iii) synthesising this new knowledge alongside the full range of changes projected by the CMIP climate models.

The approach of HyCRISTAL was to assume any projection from a globally recognised climate model was plausible until proven otherwise through analysis of the realism of climate change relevant features (see discussion on process-based evaluation above), and to recognise that the actual future could always lie outside the range produced by climate models. 


\section{CONCLUSION}

It is clear that with concerted research and funding, supported by advances in computing capacity and observational platforms, our understanding of the climate system and ability to simulate its behaviour is advancing rapidly. This is important and valuable in building confidence in model projections of future change.

However, this chapter and the experiences of many others show that advances in climate science do not effortlessly translate into improved decision-making. The dominant narrative to address this challenge is the need for improved translation, communication and co-production approaches. This speaks to the imperative that climate science is producing information that is relevant to real-world decision contexts-we argue that an effective distillation process that opens up for deliberation with all stakeholders, the wide range of value judgements and choices made within climate science, is also crucial to avoid poor decision-making as well as building trust and ownership of information.

The key principles of climate information distillation we can identify through the experience of the case studies described amongst others can be summarized as follows:

- Develop an understanding of the decision context, not just the general problem area (e.g. water resources), but also the decision space, the options, by whom and how decisions are made. This understanding can strongly inform approaches to constructing climate evidence, characterising uncertainty and avoiding risks of concern.

- Similarly, adopt a contextual risk-framing approach that allows the concerns and risks of the context experts to guide and frame the construction of climate information.

- Understand how uncertainty influences the decision. Through approaches like scenario planning and decision scaling, understand how uncertainty may challenge decision-making.

- Use climate evidence (observations, model projections, downscaling) that clearly adds value to the decision. Simplicity facilitates common understanding and engagement. The added value of the newest models and results takes time to evaluate and should not be assumed.

- If there remains a desire to reduce uncertainty by excluding implausible futures, pursue this transparently and with open deliberation about the potential risks of error. 
- Focus on the process and building trust and common understanding across climate and contextual experts. Trust and understanding are important factors behind information uptake and application.

In this chapter, we have tried to push the dominant narrative on information use further back into the climate science process itself and, in so doing, raised important questions about the many assumptions and value judgements that are made within the climate science domain prior to or during the generation of information. These assumptions, many of which are conventionally considered disciplinary and technical, nevertheless have significant implications for the resultant information and risk management decisions. Ultimately, decision-making is a process of risk management and information is used to avoid error. By adopting a more transparent, open, and deliberative distillation process, information can be constructed that integrates the value judgements and understanding of risk and uncertainty of all stakeholders, rather than just climate scientists.

\section{REFERENCES}

African Climate Risks Conference. (2019). African climate risks conference. Retrieved from https://www.africanclimaterisksconference2019.org/

Burgin, L., Walker, G., Cornforth, R., Rowell, D., Marsham, J., Semazzi, F., Sabiiti, G., Ainslie, A., Araujo, J., Ascott, M., Clegg, D., Clenaghan, A., Lapworth, D., Lwiza, K., Macdonald, D., Petty, C., Seaman, J., \& Wainwright, C. (2019). FCFA HyCRISTAL climate narrative rural infographic and brief. https://doi.org/10.5281/zenodo.3257288

Daron, J., Burgin, L., Janes, T., Jones, R. G., \& Jack, C. (2019). Climate process chains: Examples from Southern Africa. International Journal of Climatology, 39(12), 4784-4797.

Dosio, A., Jones, R. G., Jack, C., Lennard, C., Nikulin, G., \& Hewitson, B. (2019). What Can We Know About Future precipitation in Africa? Robustness, significance and added value of projections from a large ensemble of regional climate models. Climate Dynamics, 53(9-10), 5833-5858.

Eyring, V., Bony, S., Meehl, G. A., Senior, C. A., Stevens, B., Stouffer, R. J., \& Taylor, K. E. (2016). Overview of the coupled model intercomparison project phase 6 (CMIP6) experimental design and organization. Geoscientific Model Development, 9, 1937-1958. https://doi.org/10.5194/gmd-9-1937-2016.

Finney, D., Marsham, J., Rowell, D., Way, C., Evans, B., Cornforth, R., HoughtonCarr, H., Mittal, N., Allan, R., Anande, D., \& Anyah, R. (2019). Scientific understanding of East African climate change from the HyCRISTAL project. 
Finney, D. L., Marsham, J. H., Rowell, D. P., Kendon, E. J., Tucker, S. O., Stratton, R. A., \& Jackson, L. S. (2020). Effects of explicit convection on future projections of mesoscale circulations, rainfall, and rainfall extremes over Eastern Africa. Journal of Climate, 33(7), 2701-2718.

Giorgi, F. (2020). Producing actionable climate change information for regions: The distillation paradigm and the 3R framework. The European Physical Journal Plus, 135(6), 435.

Harold, J., Coventry, K., Lorenzoni, I., Kavonic, J., Diop, I. S., \& Visman, E. (2019). Improving methods of communicating climatic uncertainties to aid decisionmaking: Project report and guidelines prepared for future climate for Africa. Retrieved from http://www.fractal.org.za/wp-content/uploads/2019/02/ FCFA-Report-Communicating-Climate-Change-to-Decision-Makers.pdf

Hawkins, E., \& Sutton, R. (2011). The potential to narrow uncertainty in projections of regional precipitation change. Climate Dynamics, 37(1-2), 407-418.

Hooker, J., Duveiller, G., \& Cescatti, A. (2018). A global dataset of air temperature derived from satellite remote sensing and weather stations. Scientific Data, $5(1), 1-11$.

Jack, C., \& Katragkou, E. (2019). Evaluation of downscaling methods over Europe: Results of the EU-COST action VALUE. International Journal of Climatology, 39(9), 3689-3691.

Jack, C., Jones, R., Burgin, L., \& Daron, J. (2020). Climate risk narratives: An iterative reflective process for co-producing and integrating climate knowledge. Climate Risk Management, 29, 100239. https://doi.org/10.1016/j. crm.2020.100239.

Jackson, L. S., Finney, D. L., Kendon, E. J., Marsham, J. H., Parker, D. J., Stratton, R. A., Tomassini, L., \& Tucker, S. (2020). The effect of explicit convection on couplings between rainfall, humidity and ascent over Africa under climate change. Journal of Climate, 33, 8315.

James, R., Washington, R., Abiodun, B., Kay, G., Mutemi, J., Pokam, W., Hart, N., Artan, G., \& Senior, C. (2018). Evaluating climate models with an African lens. Bulletin of the American Meteorological Society, 99(2), 313-336.

Kendon, E. J., Stratton, R. A., Tucker, S., Marsham, J. H., Berthou, S., Rowell, D. P., \& Senior, C. A. (2019). Enhanced future changes in wet and dry extremes over Africa at convection-permitting scale. Nature Communications, 10, 1), 1-1), 14.

Knutti, R., \& Sedláček, J. (2013). Robustness and uncertainties in the new CMIP5 climate model projections. Nature Climate Change, 3(4), 369.

Knutti, R., Masson, D., \& Gettelman, A. (2013). Climate model genealogy: Generation CMIP5 and how we got there. Geophysical Research Letters, 40(6), 1194-1199. 
Mastrandrea, Michael D., et al. (2011). The IPCC AR5 guidance note on consistent treatment of uncertainties: A common approach across the working groups. Climatic Change, 108, 675.

McClure, A. (2020). Inclusive, participatory and reflexive learning processes for climate resilience: Key lessons from FRACTAL. FRACTAL Working Paper 9. Retrieved from http://www.fractal.org.za/wp-content/uploads/2020/04/ FRACTAL-learning-workin-paper_layout.pdf

McSweeney, C. F., Jones, R. G., \& Booth, B. B. (2012). Selecting ensemble members to provide regional climate change information. Journal of Climate, 25(20), 7100-7121.

Munday, C., \& Washington, R. (2019). Controls on the diversity in climate model projections of early summer drying over Southern Africa. Journal of Climate, 32(12), 3707-3725.

Parker, W. S. (2013). Ensemble modeling, uncertainty and robust predictions. Wiley Interdisciplinary Reviews: Climate Change, 4(3), 213-223.

Rowell, D. P. (2019). An observational Constraint on CMIP5 projections of the East African long rains and Southern Indian Ocean warming. Geophysical Research Letters, 46(11), 6050-6058.

Rowell, D. P., Booth, B. B., Nicholson, S. E., \& Good, P. (2015). Reconciling past and future rainfall trends over East Africa. Journal of Climate, 28(24), 9768-9788.

Rowell, D. P., Senior, C. A., Vellinga, M., \& Graham, R. J. (2016). Can climate projection uncertainty be constrained over Africa using metrics of contemporary performance? Climatic Change, 134(4), 621-633.

Sherwood, S. C., Bony, S., \& Dufresne, J. L. (2014). Spread in model climate sensitivity traced to atmospheric convective mixing. Nature, 505(7481), 37-42.

Stratton, R. A., Senior, C. A., Vosper, S. B., Folwell, S. S., Boutle, I. A., Earnshaw, P. D., Kendon, E., Lock, A. P., Malcolm, A., Manners, J., \& Morcrette, C. J. (2018). A Pan-African convection-permitting regional climate simulation with the met office unified model: CP4-Africa. Journal of Climate, 31(9), 3485-3508.

van Oldenborgh, G. J., Collins, M., Arblaster, J., Christensen, J. H., Marotzke, J., Power, S. B., Rummukainen, R., Zhou, T., \& Qin, D. (2013). Annex I: Atlas of global and regional climate projections. In IPCC Climate change 2013: The physical science basis (pp. 1311-1394).

Wilks, D. S. (2011). Statistical methods in the atmospheric sciences (Vol. 100). Oxford: Academic Press. 
Open Access This chapter is licensed under the terms of the Creative Commons Attribution 4.0 International License (http://creativecommons.org/licenses/ by $/ 4.0 /)$, which permits use, sharing, adaptation, distribution and reproduction in any medium or format, as long as you give appropriate credit to the original author(s) and the source, provide a link to the Creative Commons licence and indicate if changes were made.

The images or other third party material in this chapter are included in the chapter's Creative Commons licence, unless indicated otherwise in a credit line to the material. If material is not included in the chapter's Creative Commons licence and your intended use is not permitted by statutory regulation or exceeds the permitted use, you will need to obtain permission directly from the copyright holder. 